\title{
Vom Analogen zum Digitalen und zurück
}

\section{Zur technischen Geste}

Zusammenfassung: Dieser Artikel untersucht, wie der Umgang mit technischen Objekten von einfachen Werkzeugen bis hin zu smart devices durch Gesten bestimmt wird. Einerseits sind technische Objekte durch Gesten ,manipulierbar‘, die an, in und auf ihnen Anwendungen finden: Operationen werden durch Gesten mit technischen Objekten (im Falle des Werkzeugs) möglich oder in technischen Objekten (im Falle einer Maschine, beispielsweise eines Motors) ausgelöst. Diese Operationen sind das Resultat menschlicher Erfindung, die andererseits eine menschliche Geste in einer materiellen Struktur (dem technischen Objekt) fixiert und kristallisiert. Technische Prozesse zeigen jedoch auch, dass es „natürliche, menschliche“ Gesten im emphatischen Sinne nicht gibt. Menschliche Gesten sind immer sozio-technisch geformt, da sie Operationen zwischen dem Menschen und der Umwelt vermitteln. Die Einführung des smart phones, die gerade einmal zehn Jahre zurückliegt, illustriert, inwiefern sich das Verhältnis des Menschen zur Maschine transformiert hat. Zwar kehren Gesten zurück und werden auf der Oberfläche der Objekte eingesetzt, um diese als Werkzeuge zu benutzen, aber die Gesten liefern weder einen unmittelbaren Zugang zum technischen Objekt, sondern verbleiben notwendig auf der Oberfläche. Es zeigt sich vielmehr, inwiefern das technische Objekt auf den Menschen zurückwirkt: Die Gesten werden ausgeführt, um das smart phone logische Operationen ausführen $\mathrm{zu}$ lassen, die notwendig verborgen bleiben. Mentale und körperliche Operationen verschachteln sich ineinander und wirken wechselseitige aufeinander. Um eine ethische und symmetrische Beziehung zur Technik herzustellen, ist daher ein pädagogisch-aufklärerischer Akt notwendig, der den Dualismus des Digitalen zwischen Körper und Geist unterläuft, um das „Denken mit den Händen“ für das digitale Zeitalter wiederzuentdecken.

\section{Einleitung}

Der Kontakt mit technischen Objekten, von Werkzeugen, Geräten, Instrumenten bis hin zu smart devices, wird wesentlich durch den Einsatz von Gesten bestimmt. Diese Gesten erlauben es, technische Objekte zu benutzen, um einen bestimmten Zugang zur Welt zu gewinnen. Als Medium haben die technischen Objekte dabei einen eigentümlichen Status: Einerseits sind sie durch Gesten ,manipulierbar, 
d.h. durch Gesten, die an, in und auf ihnen Anwendungen finden, werden Operationen mit technischen Objekten (im Falle des Werkzeugs) oder in den technischen Objekten (im Falle einer Maschine, beispielsweise eines Motors) möglich und ausgelöst. Diese Operationen sind das Resultat menschlicher Erfindung, die andererseits eine menschliche Geste in einer materiellen Struktur (dem technischen Objekt) fixiert und kristallisiert (Simondon 2012, 127). Technische Objekte sind weder das Resultat von Organprojektion (Kapp 2015) noch künstliche Organe (Bergson 1933 [1932], 309), sondern resultieren aus der Fixierung der Geste in einer materiellen Struktur, die unabhängig vom menschlichen Körper existiert und dadurch eine eigene Seinsweise besitzt.

Technische Prozesse zeigen jedoch auch, dass es ,natürliche, menschliche‘ Gesten im emphatischen Sinne nicht gibt. Menschliche Gesten sind immer soziotechnisch geformt, da sie Operationen zwischen dem Menschen und der Umwelt vermitteln. Die technischen Gesten, die mit technischen Objekten aller Art vom Faustkeil bis zum Tablet ausgeführt werden, sind „,ausgestattete Handlungen“ (action outillé) ${ }^{1}$ (Sigaut 2012, 7), die zudem als „traditionelle, wirksame Handlungen“ historisch tradiert werden (Mauss 2010, 205). Technische Gesten sind daher sozio-technische Phänomene par excellence: Sie erfordern, dass sie als operationalisiertes Wissen von Autoritäten weitergegeben werden (ebd., 203). Ihr sozio-technischer Charakter unterläuft die Trennungen zwischen Natur und Kultur sowie zwischen Geist und Materie, indem sie eine geteilte Aufmerksamkeit (attention partagé) zwischen Werkzeug und menschlichem Akteur ins Werk setzen (Sigaut 2012, 132). Erst indem der Mensch sich dem Werkzeug unterwirft und seine Gesten ihm anpasst, kann das Werkzeug dem Menschen dienen (ebd.). Das Verhältnis des Menschen zum Werkzeug ist daher wesentlich rekursiv. Es ermöglicht den Zugang zur Materie, die wiederum nicht beliebig formbar ist, sondern die technische Operation mitprägt: Die Materialien der Ziegelsteinherstellung können beispielswiese nicht beliebig gewählt werden, sondern müssen bestimmte Eigenschaften aufweisen, damit sich der Ziegel, nachdem er gebrannt wurde, von seiner Form lösen lässt (Simondon 2005, 39 ff.). ${ }^{2}$

1 „Ausgestattet“ als Übersetzung für „outillée“ gibt nur unzureichend wieder, dass in der Geste bereits etwas Werkzeughaftes steckt, da „outil“ bekanntlich im Französischen für das Werkzeug steht.

2 Diese Auffassung findet sich bereits bei Mauss und wird sowohl von Simondon als auch von Sigaut geteilt (Mauss 2015; Sigaut 2012, 100; Simondon 2005, 56 f.). In dieser Hinsicht haben der sogenannte ,material turn“ und die Positionen des ,new materialism‘ langen Vorlauf. 
Aber wie weit trägt diese These? Kann man eine an Gesten orientierte Technologie ${ }^{3}$ auf Maschinen und Algorithmen anwenden, die Maschinen steuern? Werden Maschinen und Algorithmen durch Gesten zu natürlich-künstlichen Objekten? Die folgenden Ausführungen bestehen in dem Versuch, ein Spannungsfeld zwischen der Geste als ,action outillée' und dem technischen Objekt als kristallisierter Geste zu eröffnen, um die Frage zu stellen, wie sich der Kontakt mit modernen ,smart devices' und die Teilhabe an der digitalen Welt gestaltet. Die klassische Trennung zwischen Natürlichkeit und Künstlichkeit wird dabei unterlaufen. Gesten sind bereits Teil eines technischen Prozesses und können daher nicht als natürliche Phänomene gelten. Technische Objekte wiederum sind Ausdruck - so eine zentrale These der französischen Tradition der Technikanthropologie - des Lebens. Es geht dieser Tradition jedoch keineswegs darum, die menschliche Bedingung im Sinne des Transhumanismus hinter sich zu lassen, sondern vielmehr die Technik als sine qua non des Menschen (wieder) zu entdecken (Hussain 2018).

Zunächst werde ich die technische Geste vor dem Hintergrund des Begriffs der Operationskette diskutieren. Hierzu werde ich Sigauts Begriff der ,action outillée als Ausdruck einer von Mensch und technischem Objekt geteilten Aufmerksamkeit ins Zentrum stellen.

Die Frage nach bestimmten Techniken des Körpers, die mit technischen Objekten geschaffen werden, gewinnt hierbei eine besondere Bedeutung. Je nach Ebene lassen sich unterschiedliche Verhältnisse des menschlichen Akteurs und seines Körpers zur technischen Operation unterscheiden: Ist die technische Operation im Handwerk unmittelbar, wird die Distanz, je industrialisierter die technischen Objekte werden, zwischen Körper und Maschine größer. Maschine und menschlicher Körper emanzipieren sich voneinander.

Dieses Verständnis der Geste wird durch das angestrebte erkenntnistheoretische Verhältnis zur Technik bei Gilbert Simondon erweitert: Die kristallisierten

3 Technologie als zentrale Wissenschaft der Anthropologie geht auf die Debatte um den Homo faber, die sich an der Veröffentlichung Bergsons „Schöpferischer Evolution“ 1907 entzündete und bis in den zweiten Weltkrieg hinein geführt wurde (Sigaut 2018; Schick 2018), zurück. Daniel Parrochia verbindet mit dem Begriff des ,homo faber eine Reaktion auf eine positivistisch orientierte Technikphilosophie (Parrochia 2009, 53). Dies drückt sich im spezfischen Unterscheidungsmerkmal der französischen Technikphilosophie, das Loeve, et al. im jüngst erschienen Buch „French Philosophy of Technology“ festhalten, aus: „One major distinctive feature of these French approaches to technology compared to other twentieth-century traditions of the philosophy of technology is the rejection of a functionalist view of technology and, instead, an effort to understand and to evaluate technology per se. Tools, objects, machines, operations, and gestures, are scrutinized for their own sake rather than as means for external ends or for the purpose of the moral evaluation of these ends.“ (Loeve/Guchet/Bensaude Vincent 2018, 7) 
Gesten in industriell gefertigten Maschinen und Maschinen zur industriellen Fertigung stehen nicht mehr als Werkzeuge in direktem Kontakt mit dem Menschen, sondern bilden technische Ensembles, d.h. sie stehen in Relation zueinander. Der Mensch hat seit der ersten industriellen Revolution nur Zugang zu diesen technischen Ensembles, indem technische Elemente durch Werkzeuge repariert, ausgetauscht oder verändert werden. Das Verhältnis zur technischen Realität transformiert sich daher radikal, indem es sich aufspaltet: Der Mensch ist entweder auf Ebene des Elements aktiv - d. h. auf der Ebene der Einzelteile - oder greift auf der Ebene des Ensembles ein, um die technischen Objekte zu nutzen, d.h. dort, wo die technischen Objekte instrumentalisiert werden und nicht ihre eigentümliche Funktionsweise im Vordergrund steht. Es entsteht folglich, wie Pierre Charrié treffend feststellt, ein Konflikt zwischen Maschine und Geste: Die in der Maschine eingelagerten Gesten haben das Potential, jene Gesten zu ersetzen, die vorher für den Körper reserviert waren und wirken rekursiv auf den Menschen zurück, d.h. sie sind Ausgangspunkt für neue Gesten, Körpertechniken und soziale Rhythmen (Charrié 2008, 10f.).

Die Einführung des ,smart phones‘, die gerade einmal zehn Jahre zurückliegt, illustriert, inwiefern sich das Verhältnis von Mensch und Maschine transformiert hat. Zwar kehren Gesten zurück und werden auf der Oberfläche der Objekte eingesetzt, um diese als Werkzeuge zu benutzen, aber die Qualität der eingesetzten Gesten hat sich erneut verändert und verschoben: Die Gesten liefern weder einen unmittelbaren Zugang zum technischen Objekt, wie im Falle des Handwerkzeuges, noch erlauben die körperlich ausgeführten Gesten eine Manipulation des technischen Objekts, sondern verbleiben notwendig auf der Oberfläche. Vielmehr zeigt sich anhand der ,smart devices', inwiefern das technische Objekt auf den Menschen zurückwirkt: Die Gesten werden ausgeführt, um das technische Objekt logische Operationen ausführen $\mathrm{zu}$ lassen, die notwendig verborgen bleiben, aber selbst mentale Gesten sind, die in technischen Objekten kristallisiert wurden. Unter mentalen Gesten verstehe ich in diesem Zusammenhang logische Operationen, wie den Satz des Widerspruchs oder den Satz vom ausgeschlossenen Dritten, die materialisiert werden können. Ein Beispiel ist das Motherboard eines jeden Computers: Dort werden Kippschalter aneinandergereiht, die entweder offen oder geschlossen, im Zustand 0 oder 1 sind. Dies ist eine Materialisierung der ,mentalen Geste، des Satz vom ausgeschlossenen Dritten: der Kippschalter kann nur entweder geschlossen oder offen sein. Nun kann man zurecht fragen, ob der Begriff der Geste damit nicht über die Maße strapaziert wird und ob damit nicht begriffliche Schärfe verloren geht. An diese Ausweitung des Gestenbegriffs ist eine systematische These geknüpft, die die Grundlage für die Argumentation dieses Artikels liefert: Praxis ist der Kognition vorgelagert. Dies bedeutet, dass selbst logische Operationen auf Praktiken zurückgehen, die das 
Lebewesen Mensch ausführt, um sich Welt zu erschließen (Bergson 2013, 162; Simondon 2005, 529f.). Dies führt jedoch keineswegs in einen naiven Realismus, der alles Geistige auf Materielles reduziert, sondern zeigt, inwiefern das menschliche Denken und Handeln in der Welt verflochten ist. ${ }^{4}$

Mentale Gesten und körperliche Gesten verschachteln sich ineinander und bilden transduktive Operationsketten. Allerdings ist das Schicksal der technischen Objekte keineswegs durch die kausale Aneinanderreihung vorherbestimmt, sondern bleibt aufgrund der gegenseitigen Durchdringung des Sozialen und des Technischen ein „freies Abenteuer“ (Simondon 2014a, 28). Um eine Beziehung zur Technik herzustellen, ist daher ein pädagogisch-aufklärerischer Akt notwendig, der den Dualismus des Digitalen zwischen Körper und Geist unterläuft und das „Denken mit den Händen“ (Mauss 1933, 119) für das digitale Zeitalter wiederentdeckt.

\section{Geste und geteilte Aufmerksamkeit}

François Sigaut formuliert in „Comme Homo devint faber“ eine umfangreiche Analyse menschlicher Gesten, die deshalb notwendig wird, weil mehr Wissen über den gestischen Apparat der Primaten als über menschliche Gesten vorhanden ist (Sigaut 2012, 7).

Um Techniken und technische Objekte $\mathrm{zu}$ bestimmen, wurde seit Mauss versucht, diese zunächst zu klassifizieren, um sie dann identifizieren zu können. Eine Konsequenz dieser Gleichsetzung ist, dass Werkzeuge auf einen Archetyp zurückgeführt werden und so die Unterschiede zwischen Werkzeugen und den damit verbundenen Gesten verloren gehen. ${ }^{5}$ Zwar fokussiert sich André LeroiGourhan bereits in seinen beiden ersten Werken zur Technik „L'homme et la matière“ (1943) und „Milieu et technique“ (1945) auf menschliche Gesten und entwirft umfangreiche Klassifikationsschemata für Gesten, die mit Werkzeugen ausgeführt werden können. Laut Sigaut begehen die klassifikatorischen Ansätze den Fehler, Klassifikation mit Identifikation gleichzusetzen (Sigaut 2012, 38): Die Unterschiede zwischen verschiedenen „Techniken des Körpers“ können zwar

4 Ich verweise hier auf Timothy Ingolds Begriff des, entanglement', der die Beziehungen des Lebewesen zu sich selbst und mit seinen Umwelten bezeichnet (Ingold 2011).

5 „Thus Leroi-Gourhan [...] felt confident enough to write that 'the palaeontology of the knife can be traced back without a break to the first tools' [...]. This statement assumes that there is only one type of knife (our own), of which others are simply more or less incidental variations." (Sigaut 1994, 430) 
festgestellt werden, aber, so konstatiert Sigaut, das Problem der Bedeutung dieser Unterschiede ist immer noch ungelöst (ebd., 40).

Leroi-Gourhan illustriert anhand des Begriffs der „Operationskette“ (chaîne opératoire) den wesentlichen (sozio-)technischen Vorgang der Genese von Handlungsprogrammen, der die Trennung von Instinkt und Intelligenz unterläuft. Operationsketten zeigen unterschiedliche Komplexitätsgrade von Prozessen an, die aber alle auf eine Verkettung von Handlungen zurückgehen. Es scheint jedoch, als ob die Exteriorisierung mit Notwendigkeit aus der menschlichen Natur hervorgeht und die Richtung der Evolution - mit dem Menschen an ihrer Spitze bereits festgelegt sei.

Operationsketten basieren, so scheint es, auf einer These, die Sigauts Annahme entgegengesetzt ist: „Das Werkzeug ist an die Geste angepasst und nicht umgekehrt.“ (Haudricourt 1987, 158) ${ }^{6}$ Dieser Satz darf aber nicht als eine Leugnung der Reziprozität von Geste und Werkzeug verstanden werden (Schüttpelz 2006, 9). Vielmehr wird deutlich, dass sich die Fragerichtung von Leroi-Gourhan und Haudricourt hin zu Sigaut verschiebt: Gesten werden nicht mehr als Ausgangspunkt von Exteriorisierungen verstanden, die das Werkzeug formen, sondern als Möglichkeiten, wie der Körper sich dem Material anpassen kann und wie sich das Material dem Körper anpasst: d.h. es gibt keine reine Exteriorisierung eines menschlichen Inhaltes, der sich unilateral äußert. Um mit Erhard Schüttpelz zu sprechen: „Die [...] ,Exteriorisierungen“ haben nie stattgefunden.“ (ebd., 8) Weder festgelegtes Programm, das exteriorisiert werden kann, noch absoluter Ausgangspunkt des Programms können festgestellt werden. Die technische Operation selbst unterläuft bereits die Trennung zwischen Geste und Werkzeug. Beides bildet sich erst im Zusammenspiel aus.

Der rekursive Prozess der Ausbildung von Techniken zwischen Mensch und Werkzeug, kann - im Ausgang von Mauss, Leroi-Gourhan und Haudricourt - mit der Vorgängigkeit der Operationsketten thematisiert werden. ${ }^{7}$ Dies bedeutet aber nicht, dass aufgrund dieser Vorgängigkeit begriffliche Unterscheidungen zwischen Handlungen, Operationen und technischen Objekten aufgegeben werden müssen, sondern dass es immer schon ein historisch-praktisches Milieu gegeben hat, das die Entstehung von Operationen, Gesten, Werkzeugen - oder allgemeiner Individuationen - überhaupt zulässt.

6 „L'outil est adapté au geste et non inversement.“ Alle Übersetzungen aus dem Französischen habe ich, wenn nicht anders gekennzeichnet, vorgenommen.

7 Die These der Vorgängigkeit der Operationsketten wird innerhalb der (deutschen) Medienwissenschaften durchaus kontrovers diskutiert (siehe hierzu: Heilmann 2016; 2017, Mersch 2016, Schüttpelz 2006; 2017). 
Insgesamt lässt sich die Kritik Sigauts in drei Thesen zusammenfassen: 1 . Menschliche Gesten werden nicht als eigenständige Gesten erkannt und untersucht, sondern aufgrund evolutionärer Kontinuität auf Gesten der Primaten reduziert. Exemplarisch für diese These steht Leroi-Gourhan, der argumentiert, dass prinzipiell bereits alles beim Primaten vorhanden ist und die Gesten lediglich nach und nach „ausgeschwitzt“ werden. ${ }^{8}$ 2. Die Rekursivität menschlichen Handelns, also die Wirkung der Werkzeuge und Maschinen auf die Gesten, wird aufgrund der Externalisierung vernachlässigt oder überhaupt nicht berücksichtigt. Die Geste formt nicht nur das Werkzeug, sondern das Werkzeug formt auch die Geste. 3. Die Klassifikation von Techniken wurde mit der Identifikation von Techniken gleichgesetzt.

Sigaut hingegen formuliert vier wesentliche Elemente der Herausbildung technischer Gesten des Menschen: (1) geteilte Aufmerksamkeit (attention partagé), (2) geteilte Erfahrung (partage de l'expérience), (3) Freude am Gelingen (plaisir de réussite) und (4) Austausch von Waren und Fähigkeiten zwischen den Geschlechtern (échange entre les sexes) (Sigaut 2012, 187). ${ }^{9}$ Zwar bilden alle vier

8 „Vom Primaten zum Menschen erfahren die Greifoperationen keine wesentliche Veränderung, sie erlangen lediglich eine größere Vielfalt in den Zielen und in der Feinheit ihrer Ausführung [...] Besser noch als in jeder anderen Untersuchung wird in einer Analyse der technischen Geste deutlich, daß das heutige Gehirn des Menschen seine letzte Errungenschaft ist, denn die technischen Ergebnisse haben keinerlei Voraussetzungen in der osteo-muskulären Ausstattung, die nicht auch schon die höheren Affen besäßen: worauf es ankommt, ist der Nervenapparat. “(LeroiGourhan 2000, 299) und „In einem der vorangegangenen Kapitel sind wir zu dem Eindruck gelangt, der Mensch habe das Werkzeug im Verlauf seiner Evolution in gewisser Weise ausgeschwitzt. Der gleiche Eindruck drängt sich bei der Analyse der technischen Geste auf, ja er ist noch stärker, denn hier sehen wir das Werkzeug buchstäblich aus den Zähnen und den Nägeln der Primaten hervorgehen, ohne daß irgend etwas in der Geste den Bruch bezeichnete. [...] Der menschliche Wert der Geste liegt also nicht in der Hand, deren hinreichende Bedingung in ihrer Freiheit während der Fortbewegung besteht, sondern einzig im aufrechten Gang und in dessen paläontologischen Konsequenzen für die Entwicklung des Hirnapparates. Die fortschreitende Entfaltung der taktilen Sensibilität und des neuro-motorischen Apparates hat qualitative Bedeutung und führt zu keiner wesentlichen Veränderung der Grundausstattung.“ (ebd., 301)

9 Sigauts These wird durchaus davon geleitet, einen wesentlichen Unterschied zwischen Tier und Mensch auf Grundlage des Technischen zu formulieren. Letztlich geht es ihm jedoch darum, zu verstehen, inwiefern sich die Genese der menschlichen Gesellschaft erklären lässt. Er steht damit in der Tradition der Durkheimschule, auf die er sich auch explizit beruft. Die Unterscheidung von Mensch und Tier wird aber schlussendlich nicht aufgrund ihres technischen Vermögens getroffen, sondern weil Menschen die einzigen Säugetiere - mit Ausnahme des Nacktmulls (Heterocephalus glaber) - sind, deren Individuen spezialisierte Aufgaben besitzen und Werkzeuggebrauch aufweisen (Sigaut 2012, 172f.). Diese Arbeitsteilung gibt es natürlich bereits auf der Ebene der Insekten, diesen fehlt aber wiederum der Werkzeuggebrauch im strengen Sinne. Sigaut hebt meines Erachtens nicht auf eine spezifische Differenz ab, sondern ist an spezifischen Differenzen im 
Elemente, die Sigaut nennt, ein unteilbares Ganzes, aber uns interessiert hier insbesondere die Bedeutung der geteilten Aufmerksamkeit für das Verhältnis des Menschen zu den technischen Objekten, die ihn umgeben. Die Erfindung eines technischen Objekts vollzieht sich, folgt man Sigaut und auch Simondon, indem ein zunächst gedachtes, intellektuelles Schema materialisiert wird. Der Materialisierungsprozess selbst wirkt dabei aktiv auf das intellektuelle Schema zurück. ${ }^{10}$ Die Vermittlungsrolle zwischen den beiden Polen, Denken und materielles Objekt, spielen dabei die zum Material gewordene Materie und das Werkzeug sowie die Gesten, die das Material formen. Gesten und Materie transformieren sich im Prozess der Erfindung des Werkzeugs. Materie (hyle) und Form (morphe) bedingen sich gegenseitig. Die Plastizität des Denkens ist körperlich. Die Dinge und die körperliche Aktivität formen ebenso den Geist, wie der Geist den Körper formt (Malafouris 2013; 2015). Sobald der menschliche Körper in diesem Prozess verwickelt ist, dient er sich dem Werkzeug an, damit es dem Menschen dienen kann:

Das Werkzeug hat eine spezifische Wirkweise, da es das tut, was ich mit meinem eigenen Körper nicht vermag. Aber da es unbelebt ist, handelt es nicht von selbst, ich muss es aktivieren. Und ich muss es auf eine bestimmte Weise bedienen, die dem entspricht, was es ,will‘. Oder anders ausgedrückt, das Werkzeug dient mir, solange ich ihm auch diene [...]. In seinen gewöhnlichen Handlungen kann das Tier seine ganze Aufmerksamkeit auf das Ziel richten, das es verfolgt, da es nur seine eigenen Organe und die entsprechenden Automatismen mobilisiert. In der Werkzeug-Aktion ist dies nicht mehr möglich, denn das Werkzeug zwingt mich zu einem neuen Handeln, das dem Repertoire meiner angeborenen Automatismen fremd ist. Das Werkzeug ist nur ein Mittel, aber es drängt sich mir mit ebenso viel Kraft auf, als ob es ein Ziel wäre; es wird gewissermaßen zu einem zweiten Ziel. Der Umgang mit dem Werkzeug erfordert von mir anhaltende Aufmerksamkeit, aber ich kann meine Aufmerksamkeit nicht von dem Ziel ablassen, das ich verfolge. Das zwingt mich, meine Aufmerksamkeit zwischen den beiden zu teilen. (Sigaut 2012, 132) ${ }^{11}$

\footnotetext{
Allgemeinen und in ihrer Pluralität interessiert. Es geht nicht darum, eine bottom up oder top down Hierarchie festzulegen, sondern den Differenzen - ganz im Bergsonschen und Deleuzeschen Sinne - in ihrer Entfaltung zu folgen.

10 Sowohl Simondon als auch Sigaut scheinen die Beschreibung des „dynamischen Schemas“ bei Bergson fortzuführen und zu adaptieren. Der Prozess der Erfindung erinnert zudem an die Phänomenotechnik Bachelards und die Art und Weise, wie Bachelard die Arbeit der Wissenschaftler_in - nämlich als Realisierungsprozess - beschreibt. Zum Einfluss von Bachelard und Bergson auf Simondon siehe: Barthélémy 2008 und Schick 2019.

11 „L'outil a un mode d'action qui lui est propre, puisqu'il fait ce que je ne peux pas faire avec mon propre corps. Mais étant inerte, il n'agit pas par lui-même, c'est moi qui dois l'actionner. Et je dois l'actionner d'une certaine façon qui corresponde à ce qu'il „,veut“.. Ou pour le dire autrement, l'outil me sert à condition que je le serve aussi. [...] Dans ses actions ordinaires, l'animal peut appliquer toute son attention au but qu'il poursuit, puisque les seuls moyens qu'il mobilise sont ses propres organes et les automatismes correspondantes. Dans l'action outillée, cela n'est plus
} 
Die Beschreibung Sigauts erinnert an Bruno Latours Handlungsprogramme: Sobald ein Akteur - in Latours berühmten Beispiel eine Waffe - hinzutritt, entstehen neue Ziele (sub goals), die vorher nicht möglich waren (Latour 1994). Der Unterschied zwischen Sigauts Beschreibung und der Perspektive Latours besteht darin, dass Sigaut sich weniger für das Handeln im Netzwerk interessiert als für eine detaillierte Beschreibung technischer Gesten. Beiden ist allerdings gemein, dass, will man ein einfaches Ziel erreichen, mehrere Ziele gleichzeitig verfolgt werden müssen, bzw. mehrere Programme gleichzeitig ablaufen. Bereits ein einfaches Beispiel zeigt die Komplexität ablaufender Handlungsprogramme: Wenn ich mit einem Hammer (A) einen Nagel (B) in die Wand schlagen will (AB), fordert der Hammer von mir, eine bestimmte Handlung auszuführen (A1: ihn auf eine bestimmte Art und Weise zu halten und zu führen), damit die Handlung des Hämmerns (A2) möglich effizient gelingt. Gleichzeitig fordert der Nagel mich auf, ein analoges Handlungsprogramm mit der anderen Hand durchzuführen: Ich muss den Nagel auf eine bestimmte Weise halten (B1), um mich nicht zu verletzen und ihn in eine bestimmte Position zu bringen, um ihn möglichst gerade in die Wand zu schlagen (B2). Sind in handwerklichen Tätigkeiten die Gesten noch direkt an den menschlichen Körper gekoppelt, der Informationen und Energie zuführt, erfordert das Bedienen von Maschinen, die eigenständig operieren, multiple, ineinander verschachtelte Operationen, die einfache Gesten in komplexe Operationen und umgekehrt übersetzen.

\section{Verschachtelte Gesten im Netzwerk}

Vor dem Hintergrund dieser Theorie der technischen Geste lässt sich die These, jedes technische Objekt enthalte eine kristallisierte menschliche Geste, besser verstehen, will man sie auch auf industrielle und post-industrielle technische Objekte anwenden. Der Unterschied zwischen kristallisierten Gesten in Motoren, Dioden, Rechenmaschinen und Steuerungssystemen wird dadurch ebenso markiert wie die Gemeinsamkeiten, die sie mit Faustkeilen, Bögen, Pflügen und Töpferscheiben besitzen.

Der Unterschied scheint zunächst offensichtlich im Wortsinne: Während wir dem Messer unmittelbar eine Geste zuordnen können, weil wir nicht nur sehen,

possible, parce que l'outil m'oblige à agir d'une façon inédite, étrangère au répertoire de mes automatismes innés. L'outil n'est qu'un moyen, mais qui s'impose à moi avec autant de force que se c'était un but ; il devient un but second, en quelque sorte. Le maniement de l'outil exige de moi une attention soutenue, sans pour autant que je puisse relâcher l'attention que je porte au but que je poursuis. Ce qui m’oblige à partager mon attention entre l'un et l'autre.“ (Sigaut 2012, 132) 
wie das Messer schneidet, sondern mit ihm eine körperliche Handlung durchführen, verdoppelt sich das Verhältnis zur Maschine, in Gesten, die an und mit der Maschine durchgeführt werden, und Gesten, die in der Maschine kristallisiert wurden, d.h. Operationen, die ihren Ursprung im Menschen hatten und nun die Basis für das Funktionieren der Maschine sind.

Der entscheidende Punkt ist hier, dass in den Maschinen nicht mehr notwendig der funktionale Aspekt - z.B. das Zerschneiden mit dem Messer, das schnelle Ausrechnen von Aufgaben durch den Computer - den Wesenskern und damit die kristallisierte Geste ausmacht, sondern die Operationalisierung und Übersetzung dieser Geste in andere Operationen, wie beispielsweise die Übertragung komplexer Vorgänge in eine Aneinanderreihung von Kippschaltern, die entweder offen (0) oder geschlossen sind (1), wie es beim Computer der Fall ist.

Die Verdopplung ist Ausdruck - folgt man Gilbert Simondon - der ontologischen Komplementarität von Operation und Struktur, die gleichzeitig in allen ,Handlungsfolgen“ (actes) vorhanden ist: Die Maschine ist Struktur gewordene Operationalität. Simondon wählt das Beispiel des Zeichnens einer Parallele, um diese Komplementarität zu beschreiben:

Wenn der Geometer also eine Parallele [...] zieht, achtet der Geometer in seiner gesamten Handlung auf das Strukturelement, das nur das geometrische Denken interessiert, nämlich die Tatsache, dass es eine Linie ist, die gezogen wird, und in welche Beziehung sie zu einer anderen Linie steht. Die Struktur des Aktes ist hier die Parallelität einer Linie zu einer anderen Linie. Aber der Geometer könnte auch auf den operativen Aspekt seines Handelns achten, d.h. die Geste, mit der er zeichnet, ohne sich Gedanken darüber zu machen, was er zeichnet. Die Geste des Zeichnens besitzt ihren eigentümlichen Schematismus. Das System, an dem sie Teil hat, ist ein operatives System und kein strukturelles System; die Geste geht von einem Willensakt aus, der selbst wiederum eine gewisse mentale Geste ist; sie setzt die Verfügbarkeit einer bestimmten Energie voraus, die durch die mentale Geste befreit und kommandiert wird und durch alle Glieder einer Kette komplexer, konditionaler Kausalitäten läuft [Hervorhebung J.S.]. Die Ausführung der Geste setzt eine interne und externe Regulation der Bewegung in einem operationalen Schema der Finalität ins Spiel. (Simondon 2005, $529 \mathrm{f}.)^{12}$

12 „Ainsi, quand le géomètre trace une parallèle [...], le géomètre prête attention, dans la totalité de son acte, à l'élément structural qui seul intéresse la pensée géométrique, à savoir le fait que c'est une droite qui est tracé, et avec telle relation avec une autre droite. La structure de l'acte est ici le parallélisme d'une droite par rapport à une autre droite. Mais le géomètre pourrait aussi prêter attention à l'aspect d'opération de son acte, c'est à dire au geste par lequel il trace sans se préoccuper de ce qu'il trace. Ce geste de tracer possède son schématisme propre. Le système dont il fait partie est un système opératoire, non un système structural ; ce geste procède en effet d'un volition qui est elle-même un certain geste mental ; il suppose la disponibilité d'une certaine énérgie qui se trouve libérée et commandée par le geste mental à travers tous les maillons d’une 
Die These der Operationsketten wird hier von Simondon aufgenommen, um zu zeigen, (1) dass alle Handlungsabläufe gleichzeitig Operationen und Strukturen enthalten, (2) dies sich bereits in elementaren Gesten wie der des Zeichnens einer Parallele zeigt und (3) inwiefern diese ,mentale Gesten“ in Operationen übersetzt werden: Statische Strukturen sind den Operationen nicht vorgängig. Strukturen werden nicht nur einfach aktualisiert, sondern hervorgebracht.

,Gesten' umfassen, wie hier deutlich wird, mehr als die rein körperlichen Handlungen. Innerhalb der französischen Tradition der Technikphilosophie wird dieser Begriff äußerst weit gefasst. Zunächst können Gesten als wesentlich kommunikativ gekennzeichnet werden (Guillerme 2017, 449). Sie drücken etwas aus und lassen sich nicht auf eine rein körperliche Bewegung reduzieren. Dies führt zu einer zunächst trivialen These: Wenn Gesten tatsächlich in Objekten eingelagert sind, dann kommunizieren diese Objekte etwas, sie haben Bedeutung und sagen etwas aus. Diese Perspektive lenkt den Blick von der reinen Anwendung des Objektes um eines anderen Zwecks willen auf dessen Entstehung und epistemologischen Status. Die teleologische Frage „welches Ziel kann ich damit erreichen?“ wird durch die Fragen ergänzt: „welche menschliche Geste ist in diesem Objekt kristallisiert?“, „was drückt dieses Objekt aus?“ und „welche Operationen führt dieses Objekt aus?“.

Es ist jedoch notwendig, die genetische Frage „wie ist dieses Objekt entstanden?“ zu stellen, um einerseits die Spezifizität technischer Objekte zu erfassen und andererseits die epistemologische Situation des menschlichen Subjekts mitzudenken. Erst durch diese erneute Änderung des Blicks gelangt das Subjekt in ein rekursives Verhältnis mit dem technischen Objekt: Es erkennt, inwiefern das technische Objekt als geschaffenes existiert, welche menschlichen Eigenschaften es in sich trägt und inwiefern es sich vom Menschen unterscheidet. Dadurch, dass wir erkennen, wie das technische Objekt als technisches Objekt funktioniert und eine menschliche Geste ausdrückt, gewinnen wir einen Zugang zur Existenzweise des Objekts, der Relation, die wir mit dem Objekt eingehen und wie dieses auf uns wirkt.

In „Die Existenzweise technischer Objekte“ betont Gilbert Simondon, dass das technische Objekt auf drei verschiedenen Ebenen adressiert werden kann und - will man ein holistisches Bild der Beziehungen des Menschen zur Technik zeichnen - muss. Die Ebenen des Elements, des Individuums und des Ensembles entsprechen dabei zwar jeweils unterschiedlichen Zugangsweisen, können aber auch nicht voneinander getrennt werden. Simondon spricht auch technischen

chaîne de causalités conditionnelles complexes. L'exécution de ce geste met en jeu une regulation interne et externe du mouvement dans un schème opératoire de finalité.“ (Simondon 2005, 529 f.) 
Objekten als Individuen, da diese aus Individuationsprozessen hervorgehen. Diese Charakterisierung hat methodische Gründe: das Ziel seiner Technikphilosophie ist, technische Objekte als kulturelle Objekte zu verstehen (Simondon 2012, $9 \mathrm{ff}$.). Er entwickelt daher eine Methodik, die Genese technischer Objekte, das heißt ihre Individuation, zu verstehen. In seiner zweiten Dissertation wendet er diese Methode an, um Individuation im Allgemeinen, von der physikalischen Ebene (bei Kristallen), bis hin zu sozialen Prozessen in Analogie zueinander zu setzen (Simondon 2005). Dabei handelt es sich um eine komplexe Ontologie der Immanenz, die auf die Potentialität des Seins in den unterschiedlichen Prozessen der Hervorbringung verweist. Genau diese Potentialität steckt auch in der Technik und ist für Simondon ein wesentlich menschlicher Zug, der auch Technik als Ausdruck lebendiger Prozesse erscheinen lässt (Simondon 2014).

Zwar verlaufen diese technischen Individuationsprozesse anders als lebendige Individuationsprozesse, aber technische Objekte durchlaufen eine ihnen eigene Evolution. Sie resultieren aus menschlichen Erfindungen und tendieren zur Organisationsform des Lebendigen - auch wenn diese nicht erreicht werden kann: während ein Lebewesen von Geburt an ein Ganzes bildet, ist ein technisches Objekt geschaffen und zusammengesetzt (Simondon 2012, 45). Die Organe des Lebewesens sind plurifunktional und stehen im wechselseitigen Austausch mit dem Körper als Ganzem. Ein technisches Individuum besteht immer aus technischen Elementen, das heißt, es bleibt zusammengesetzt und geschaffen.

Gleichzeitig ist das technische Objekt auch Teil eines Ensembles, das heißt Teil eines Zusammenschlusses verschiedener technischer Objekte, die ein Netzwerk bilden - mit Heidegger könnte man vom ,Gestell‘ reden, aber Simondon vermeidet die negative Konnotation, die die polare Gegenüberstellung von Natur und Technik des Gestells impliziert. Ensembles vermitteln vielmehr zwischen menschlichen und natürlichen Größen. Element, Individuum und Ensemble stellen jeweils unterschiedlich Perspektiven und Evolutionsstufen des Technischen dar.

Elemente sind die kleinste Einheit der technischen Objekte, die zum Gegenstand werden können. In einem technischen Objekt gibt es technische Elemente, die durch die Zeit erhalten werden können und sich auch in Weiterentwicklungen des technischen Objekts wiederfinden (zum Beispiel Schrauben). Diese Elemente sind meist das Produkt technischer Ensembles, das heißt, sie gehen beispielsweise aus industrieller Produktion hervor. Element und Ensemble verweisen also aufeinander. Ebenso ergibt es keinen Sinn, von einem technischen Ensemble zu sprechen, ohne technische Individuen $\mathrm{zu}$ implizieren. Technische Individuen stellen ein Ganzes dar, das die technische Operationsweise sichert und in sich trägt. Technische Individuen können Lebewesen involvieren, aber auch - im industriellen und post-industriellen Zeitalter - ohne den Menschen auskommen. 
Ein Pflug bildet insofern erst als Gespann mit Bäuerin und Zugochse ein technisches Individuum, da das Gespann als Ganzes die technische Operationsweise des Pflügens ermöglicht. ${ }^{13}$

Das Verhältnis zwischen technischem Element, technischen Individuum und technischem Ensemble ist historisch kontingent. Die Rolle des Menschen in dieser Konstellation wird durch die Veränderung der Produktionsbedingungen im Ensemble und der Automatisierung bestimmter technischer Operationen, die dem Menschen einst zukamen verändert. Zwar bleiben Gesten und auch technische Objekte durch die Zeit hindurch erhalten, aber die Produktionsweise und der spezifische Gebrauch sind an ein historisches Milieu gebunden, das jeweils anders sozio-technisch und natürlich gestaltet ist. Simondon illustriert dies anhand der Rolle des Menschen als ,Werkzeugträger_innen' und der Maschinen als ,Werkzeugträger‘. Im technischen Ensemble der vorindustriellen Zeit, der Werkstatt, ist der Mensch noch das technische Individuum. Die Handwerker_in verfügt über technische Elemente (z.B. Hämmer, Schrauben, Zahnräder, Getriebe), die durch die Zeit hindurch relativ stabil bleiben. Die Handwerker_in trägt die Werkzeuge und ihre Gesten werden unmittelbar durch den Prozess des Herstellens geformt.

Aber während die Handwerker_in in der Werkstatt noch die Teile, die sie zusammenbaut, und die Werkzeuge, mit denen sie dies tut, organisiert und folglich „inmitten“ der Elemente arbeitet, treten mit dem industriellen Zeitalter die Produktion der Elemente und die technische Operation auseinander. Der Mensch ist nicht mehr Werkzeugträger, wie es noch die Handwerker_in war. Das technische Individuum hat sich verändert. Diese Rolle wird nun von der Maschine übernommen (Simondon 2012, 71). Es entstehen zwei einander entgegengesetzte Perspektiven, die von oben oder von unten den technischen Prozess betrachten. Während die Arbeiter_innen nun auf der Ebene der Elemente mit den Maschinen interagieren, blickt die ,Chef_in' von oben - einem View from Nowhere - auf den technischen Prozess als Ganzes (ebd., 74). Die Arbeiter_innen reparieren und warten die Maschinen. Sie stellen ihr Funktionieren sicher, partizipieren aber nicht mehr direkt an den technischen Operationen: während die Handwerker_in noch mit Körper und Geist wesentlicher Bestandteil der technischen Operation war, beobachten die Arbeiter_innen die technische Operation auf der Ebene der Maschine von außen. Die Chef_in der Fabrik hat zwar einen Blick auf das Ganze d.h. sie hat einen Überblick von den Anfängen (Rohstoffbesorgung) bis zum Endprodukt - , lässt aber Sinn und Verständnis für die Operationen auf der elementaren Ebene der Maschine vermissen (ebd.).

13 Zur Geschichte des Pfluges siehe Haudricourt 1987. 
Nun ist das technische Individuum in diesem Falle die Maschine selbst, die als Werkzeugträger_in fungiert und die technische Operation durchführt. Der Mensch ist nicht mehr - oder nur noch in seltenen Fällen - technisches Individuum, wie er es als Handwerker_in noch war, wo er mit den Werkzeugen eine Einheit bildete und im Ensemble der Werkstatt technische Operationen durchführte. Dies ändert sich erneut im post-industriellen Zeitalter, indem der Mensch selbst auf der elementaren Ebene mit anderen technischen Objekten eine Einheit bildet, die ihn mit dem technischen Netzwerk in Beziehung setzen. Die digitalen Werkzeuge bilden eine in sich geschlossene Einheit, die über ihre Benutzeroberfläche der Nutzer_in die Möglichkeit bieten, Operationen anzustoßen, die notwendig unsichtbar bleiben und auch bleiben sollen. Die Gesten, die digitalen Operationen zu Grunde liegen (die Aneinanderreihung von Kippschaltern in offener oder geschlossener Position, 0 oder 1), werden übersetzt in Bilder, die Gesten ermöglichen, die dem Buchdruck, dem Schreiben und dem anderen Digitalen des Haltens in den Fingern- entlehnt sind: Von der mentalen Geste - dem Satz des ausgeschlossenen Dritten -, zur Repräsentation der Schrift auf dem Bildschirm dem Wahrnehmen und Erkennen von Zeichen -, dem Schreiben auf der Tastatur also aktiv Zeichen zu erzeugen -, zum Ziehen, Wischen und Zusammenführen des Daumens und Zeigefingers - also basaler, menschlicher Gesten, die auf die Befreiung der Hand zurückgehen (Leroi-Gourhan 2000), verschachteln sich Gesten ineinander und sind intrinsisch miteinander verknüpft. Einerseits besteht eine enge Beziehung zwischen Hard- und Software, andererseits offenbart und verstärkt dieser Dualismus das konkret technische Nicht-Wissen seiner Nutzer_innen. Die Nutzer_innen arbeiten zwar als technische Individuen als Hybride von Mensch und smart device, aber verbleiben auf der Ebene des Netzwerks, ohne wirklich die Operationen, die das Netzwerk ermöglichen, manipulieren zu können. Dies bleibt Experten_innen vorbehalten, die auf die Ebene der Elemente hinabsteigen, um die Übersetzung der Gesten bis in ihre Ursprünge nachzuverfolgen. Das Paradox besteht darin, dass man von einer Geste zur anderen kommen kann, aber eben nicht (mehr) muss, um technische Handlungen durchzuführen. Zwar ist die Tradition ein wesentlicher Bestandteil technischer Entwicklung man muss nicht immer alles von Neuem lernen -, sie suggeriert aber auch eine technische Überlegenheit der Moderne, die es nie gegeben hat. Die moderne „Kultur“14 hat eine Situation der Entfremdung der Arbeiter_in und des technischen Objekts hervorgebracht:

14 Um die Differenz zwischen Kultur als Gegenbegriff zur Technik und Kultur im ethnologischen Verständnis als sozio-technische Größe anzuzeigen, schreibt Simondon culture für den erstgenannten, negativen Begriff und Culture für letzteren. Diesen Unterschied kennzeichne ich durch Anführungszeichen für culture. Die Majuskel zeigt für Simondon außerdem an, dass es eine 
Das Objekt ist nicht mehr dekodierbar, nicht mehr als Resultat einer Operation der Konstruktion verständlich. Es ist fremd wie eine fremde Sprache. Man versteht unter diesen Bedingungen, warum eine solches Objekt vielleicht wie ein mechanischer Sklave behandelt wird. Man versucht nicht die Sprache des Sklaven zu verstehen, sondern möchte lediglich einen bestimmten Dienst von ihm erhalten. In der entfremdeten Situation genügen das Bedienfeld und die Bedienelemente auf dem technischen Objekt, um eine praktische Operation des Gebrauchs innerhalb einer definierten Aufgabe zu unternehmen. (Simondon 2014a, 66) ${ }^{15}$

In Ergänzung zu Marx betont Simondon, dass die Entfremdung ein Phänomen ist, das sich nicht nur auf den Wert der Arbeit bezieht, sondern auch auf der Ebene der Bedeutung Wirksamkeit entfaltet (Simondon 2014a, 55). Aus den vorangegangenen Beschreibungen geht hervor, dass unterschiedliche Gesten mit, an und auf technischen Objekten durchgeführt werden. Diese Gesten schachteln sich gleichsam ineinander und führen - je nach Blickrichtung - von der Ebene des Gebrauchs bis zur technischen Operation oder umgekehrt von der technischen Operation bis zum Gebrauch. Dies impliziert, dass - obwohl wir sehr unterschiedliche Gesten auf der Oberfläche eines technischen Objektes durchführen diese mit all den anderen Gesten, die das technische Objekt hervorgebracht haben, untrennbar verknüpft sind. Die Beziehung zwischen den Gesten der Herstellung und den Gesten des Gebrauches ist transduktiv und rekursiv. Dies bedeutet jedoch nicht, dass bereits im Ausgang gesichert ist, wie sich die Beziehung gestaltet und wie sich Gesten im Zuge der Genese entwickeln und transformieren. Die Gesten werden nämlich nicht im Sinne Leroi-Gourhans „ausgeschwitzt“, um ein Objekt zu bilden, sondern die Eigenständigkeit des technischen Objekts wirkt auf die Gesten zurück. Die ,Sprache` der Operationalität kann verlernt werden und mitunter ist dies auch so gewollt. Die Verknüpfung verschiedener Operationen über unterschiedliche Instanzen bedeutet für die Nutzer_in, für das Objekt und für die darin kristallisierten Gesten und Tätigkeiten ein freies Abenteuer:

Die Fähigkeit zur Loslösung im Ausgang vom menschlichen Operator - Künstler oder Produzent - bedeutet für den produzierten Gegenstand den Beginn eines freien Abenteuers, mit ebenso vielen Überlebens- und Übertragungschancen durch die Zeiten, wie es Gefahren der

verlorengegangene und wiedergewinnbare sozio-technische Einheit gibt, die als Ideal dienen kann.

15 „[L]'objet n'est plus décodable, plus compréhensible comme résultat d'une opération de construction. Il est étranger comme une langue étrangère. On comprend, dans ces conditions, pourquoi un tel objet peut être traité comme un esclave mécanique. On ne cherche pas à comprendre le langage de l'esclave, mais seulement à obtenir de lui un service déterminé. Sur l'objet technique en situation d'aliénation, le tableau de bord et les organes de commande suffisent à l’opération pratique de l'utilisation dans le cadre d'un travail défini.“ (Simondon 2014a, 66) 
Versklavung gibt, oder aber in einem Register grundlegender Ambivalenz, von Entfremdungsmöglichkeiten für die menschliche Tätigkeit, die in seinen Werken oder Produkten eingeschlossen und gleichsam darin kristallisiert ist. Die Arbeit domestiziert den Operator durch die Rückkehr der Effizienz, wenn sie von einem versklavten Operator oder einer versklavten operativen Geste ausgeht: Es gibt eine transduktive Beziehung und eine rekursive Kausalität in dem widerhallendem System (système réverberante), das durch den Operator, die Arbeit und die Menge der vermittelnden Realitäten zwischen dem menschlichen Operator und dem produzierten Objekt gebildet wird (Simondon 2014a, 28). ${ }^{16}$

Der wechselseitige, transduktive Übersetzungsprozess zeigt an, dass die technische Operation auf jeder Ebene einerseits Normativität erzeugt, sich aber auch für Normen öffnen kann, die nicht der technischen Operationalität entstammen. Die dreifache ,Versklavung', entweder der Gesten, der Bediener_innen oder beider, zeigt an, dass die Gesten nicht im Dienste der Operationalität stehen, sondern eine andere, der Operationalität äußerliche Norm die Gesten und/oder Bediener_innen diktiert. Sobald dies der Fall ist, treten „Kultur“ und Technik auseinander. Die vermeintliche Trennung tut so, als könne man das Soziale und das Technische trennen, um Werte zu bewahren, die durch neue Techniken gefährdet gesehen werden. Dieser konservative Aspekt der „Kultur“ entspricht einer ausschließenden Logik der In-Group, die sich jedweder Öffnung nach außen verweigert (Simondon 2005, 286). Der Status des widerhallenden Systems, das Arbeiter_innen, die Arbeit selbst, die vermittelnden Realitäten (d.h. technische Objekte) und das zu produzierende Objekt bildet, hängt davon ab, inwiefern Partizipation in diesem System möglich ist. Handelt es sich um ein geschlossenes System, so ist so wenig Partizipation wie möglich erlaubt und es wirkt „,versklavend“. Handelt es sich um ein offenes System, dann sind Offenheit und Partizipation wesentliche Prinzipien. Ein derartig konzipiertes System kann zu einer technischen Kultur führen. Es entsteht aber, sobald sich technische Operationen oder Gesten aneinanderreihen, eine axiologische Problematik, die sich in jedem Glied fortsetzt.

16 „La capacité de détachement à partir de l'opérateur humain initial - artiste ou producteur - signifie pour l'objet produit commencement d'une aventure libre, comportant autant de chances de survie et de transmissions à travers les âges que de dangers de réduction en esclavage, ou bien encore, dans un registre d'ambivalence fondamentale, de possibiliés d'aliénation pour l'activité humaine et qui est enclose et comme cristallisée dans ses œuvres ou produits. L'œuvre domestique l'opérateur par retour d'efficience quand elle émane d'un opérateur ou d'un geste opératoire asservi : il y a relation transductive et causalité recurrente dans le système réverbérante constitué par l'opérateur, l'œuvre et l'ensemble des réalités médiatrices intermédiaires entre l'homme opérateur et l'objet produit.“ (Simondon 2014a, 28) 
Es gibt zwar keine vorrangige Entität, die die Richtung vorgibt, oder ein bereits festgelegtes Ziel, das verfolgt und nur realisiert werden muss, dies bedeutet jedoch nicht, dass die Aneinanderreihung willkürlich geschieht, sondern lediglich, dass über die Operationalisierung Normativität eingeführt wird. Simondon verwendet das Beispiel der Geste des Laufens (sic!) im Wald: Bevor man zu laufen beginnt, sind alle Schritte gleichwahrscheinlich und gleichwertig, aber sobald ein Schritt unternommen wird, reiht sich der folgende Schritt an den nächsten. Impliziert ist dabei, dass das Laufen in einem bestimmten Milieu stattfindet, das sozusagen von außen ebenso wie die mentale Geste von innen das Laufen hervorbringt. ${ }^{17}$ Das Beispiel suggeriert jedoch, dass technische Gesten auf eine Umwelt angewandt werden, die ganz natürlich ist. Diesen Naturzustand gibt es aber für das menschliche Milieu und für den Prozess des Erlernens von Gesten und Körpertechniken nicht: Wie Marcel Mauss betont, werden Techniken v. a. durch die Vermittlung von Autoritäten erlernt (Mauss 2010, 203).

\section{Mit den Händen denken lernen?}

Die Entwicklung einer technischen Kultur, sei es im industriellen oder postindustriellen Zeitalter, sei es mit analogen oder mit digitalen Techniken, wird daher durch einen pädagogischen Aufwand bedingt. Dieser Aufwand wird - so hat es zumindest den Anschein - auch in den Schulen betrieben, wenn diese aufgrund von Digitalpakten auf den neuesten Stand der Technik gebracht werden sollen. Die bloße Ausstattung bereitet aber weder das Lehrpersonal noch die Schüler_innen im Sinne einer technischen Kultur auf die Herausforderungen des Digitalen vor. Vielmehr werden die immer gleichen Stereotype und Dualismen kontinuiert, anstelle eines technischen Verständnisses der Praktiken zu schaffen, die mit den digitalen Werkzeugen durchgeführt werden.

Das Argument, das hier entwickelt wird, ist dabei keineswegs technikfeindlich, sondern zielt auf die Rekursivität und gegenseitige Durchdringung des Technischen und des Sozialen, des Geistes und des Körpers sowie des Analogen und des Digitalen. Es gilt, „mit den Händen denken zu lernen“, wie Mauss sagt:

17 „Avant le geste de marcher, il n'y a pas de normes et tous les pas, en toutes les directions, sont à la fois équiprobables et équivalents. Mais dès qu'un pas est accompli, il devient norme pour le pas suivant, car le pas suivant est cumulatif par rapport à lui, et tous les pas faits dans la même direction, s'ajoutent et mènent vers la lisière de la forêt. En son origine absolue, l'acte de marcher ne comporte aucune polarité directrice, aucune norme extérieure, aucune référence à un but perçue. Le voyageur ne connaît pas la forme de la forêt, car il ne l'a pas parcourue. La norme est la dérivée de l'acte, et non une virtualité préalable qu'il faudrait actualiser.“ (Simondon 2014a, 103) 
In Marokko beispielsweise, habe ich arme, indigene Kinder ab dem Alter von fünf Jahren mit erstaunlicher Geschicklichkeit arbeiten sehen. Es handelte sich darum, Borten zu nähen; dies ist eine delikate Arbeit, die einen sicheren geometrischen und arithmetischen Sinn voraussetzt. Das marokkanische Kind ist ein Techniker und arbeitet weitaus früher als unsere Kinder. In mancherlei Hinsicht denkt es früher und schneller und auf andere Weise - mit den Händen - als die Kinder unserer guten, bourgeoisen Familien. Selbst in unseren Kindergärten verrichten unsere Kinder keine „manuelle Arbeit“ im eigentlichen Sinne, sondern spielen nur. (Mauss 1933, 119) $)^{18}$

Natürlich ist dieser Aufruf überspitzt und Mauss sollte nicht missverstanden werden, Kinderarbeit dem Spiel in unseren bourgeoisen Kindergärten, manuelle Arbeit der geistigen oder das Analoge dem Digitalen vorzuziehen. ${ }^{19}$ Es zeigt jedoch verschiedene wesentliche Momente einer möglichen technischen Kultur an: 1. Die Rekursivität der Techniken, des individuellen und des sozialen Körpers. 2. Die kulturelle Relativität der Kategorien des Denkens und Handelns. 3. Die Möglichkeit der Umgestaltung dieser Praktiken, wenn sie als solche erkannt werden.

Das „Denken mit den Händen“ besteht gerade darin, den Fokus auf die mentalen und körperlichen Gesten zu legen, die tagtäglich mit den verschiedenen digitalen, smarten Werkzeugen und Umwelten (Smartwatch, Smartphones, Smarthome, Tablets, Laptops, intelligente Fahrsysteme, etc.) durchgeführt werden. So kann zumindest die Blickrichtung nicht nur durch das Werkzeug hindurch auf die zu erfüllende Aufgabe, sondern auch auf das Objekt und die Netzwerkstruktur gelenkt werden, um die Eingebundenheit des eigenen Handelns und der daran geknüpften Werkzeuge wahrzunehmen.

Der Perspektivenwechsel wird selbstredend durch die Rolle der sozialen Autorität mitbestimmt: Wer entscheidet wie, welche Praktiken er- und gelernt werden?

Dieses Anliegen verbindet Simondon mit Mauss. Simondon, der in Schulen lehrte, entwarf ein Programm, um Schüler_innen technisches Wissen zu vermit-

18 „Il faudrait en considérer d'autres, celles d'enfants élevés dans des milieux très différents. Au Maroc, j'ai vu des enfants indigènes pauvres exercer un métier, dès l'âge de cinq ans, avec une dextérité étonnante. Il s'agissait de former des ganses et de les coudre ; c'est un travail délicat qui suppose un sens géométrique et arithmétique très sûr. L'enfant marocain est technicien et travaille bien plus tôt que l'enfant de chez nous. Sur certains points, il raisonne donc plus tôt et plus vite et autrement, - manuellement, - que les enfants de nos bonnes familles bourgeoises. Même dans nos jardins d'enfants, les élèves ne font pas de „travail manuel“ proprement dit, mais seulement des jeux. On voit donc qu'il faudrait faire des observations ethnographiques rigoureuses étendues, par exemple dans l'Afrique du Nord, avant de tirer aucune conclusion quelque peu générale.“(Mauss 1933, 119)

19 Insbesondere auch, weil das Spielen wesentlich dazu beiträgt, technische Skills auszubilden (siehe Schüttpelz im Erscheinen). 
teln (Simondon 2014b; 2014c). Seine philosophisch-pädagogische Überzeugung war, dass die Beziehung zur Technik über ein intuitives Verständnis technischer Schemata hergestellt werden kann, ohne dass die Schüler_innen zunächst verstehen mussten, was die genauen physikochemischen Vorgänge seien. ${ }^{20}$ Obwohl wesentliche Unterschiede in der Entwicklung technischer Skills zwischen verschiedenen Kulturen (hier die marokkanische der 1920er und 1930er Jahre und der französischen der 1950er und 1960er Jahre) bestehen und die Begegnung mit technischen Gesten anders gestaltet ist/wurde/wird, wachsen auch die „,bourgeoisen“ Kinder in einer Kultur auf, die die „Begegnung mit technischen Objekten“ und deshalb das Erlernen „bestimmte[r] Verhaltens- und Funktionsmuster, die technischen Ursprungs sind“, ermöglicht. Dies liefert die Basis, so Simondon weiter, um Archetypen zu entwicklen, die später eine „unersetzliche implizite und gelebte Beziehung der Vertrautheit und des intuitiven Verstehens“ ermöglichen (Simondon 2014a, 44). ${ }^{21}$

Das pädagogisch-aufklärerische Anliegen, diese Beziehung zu fördern und wiederzuentdecken, ist wesentlicher Bestandteil der Technikphilosophie Simondons. Zentral wird dabei der Begriff der Erfindung bzw. der Wiedererfindung, die einem Erlernen der weiter oben eingeführten Fremdsprache entsprechen. Gerade die Geste eines Kindes, weist, so Simondon, „mehr authentische Bildung“ auf, wenn sie „eine technische Vorrichtung von neuem erfindet als [der] Text, in dem Chateaubriand das ,erschreckende Genie‘ Blaise Pascals beschreibt. [...] Pascal zu verstehen heißt, mit seinen eigenen Händen eine Maschine wie die seine neu zu bauen, ohne zu kopieren, indem man sie gar, wenn möglich, in eine elektronische Summationsvorrichtung überführt, um etwas neu $\mathrm{zu}$ erfinden, anstatt bloß zu reproduzieren, indem die intellektuellen und operativen Schemata aktualisiert werden, die jene Pascals waren.“ (Simondon 2012, 99)

Laut Simondon wird so der Weg zu einer „technischen Kultur“ bereitet, die die philosophische Aufgabe formuliert, für das technische Objekt zu leisten, was die Aufklärung für den Begriff des Menschen geleistet hat: Einen allgemeinen Begriff des technischen Objekts zu schaffen, der ihm einen kulturellen, menschlichen Wert beimisst, oder, um die kantsche Formulierung zu verwenden, das technische Objekt als Zweck an sich zu begreifen (ebd., 9).

20 Simondon entwickelte sogar eine Klassifizierung, in welchen Jahrgangsstufen den Schüler_innen welche technischen Schemata beigebracht werden sollen (Simondon 2014a, 45f.).

21 „[N]os enfants, élevés dans une culture qui comporte la rencontre d'objets techniques, peuvent saisir certains schèmes de comportement et de fonctionnement qui sont d'origine technique, et les conserver en eux comme base d'archétypes, permettant plus tard une irremplaçable relation implicite et vécue de familiarité, de compréhension intuitive.“ (Simondon 2014a, 44) 
Das vorgestellte Modell, über die Gesten eine technische Kultur bzw. ein anderes technisches Selbstverständnis des Menschen im digitalen Zeitalter zu entwickeln, kann als hoffnungslos romantische Utopie gelesen werden. Im digitalen, postkolonialen Zeitalter wurden - gerade mit Blick auf die aktuelle politische, technische und soziale Lage - weder die Sklaverei und die Diskriminierung aufgrund von Hautfarbe oder ethnischer Herkunft, noch die Versklavung technischer Objekte vollständig beseitigt, sondern in den weltweiten „Surveillance Capitalism“ (Zuboff 2019) verschoben. Prinzipiell, so kann man nicht unberechtigt feststellen, hat sich nichts geändert in der Welt: Die Versklavung wurde globalisiert und ausgelagert, zugunsten derer, die die Produktionsmittel in der Hand halten (Scholz 2017).

Aber das kritische Potential der Technikanthropologie und einer Technologie, die auf Gesten basiert, zeigt gerade, dass, indem die unterschiedlichen Modi des Menschseins reflektiert werden, erstens die Gleichwertigkeit fremder, indigener Techniken anerkannt wird, und dass zweitens zwischen den Werten, die aus der Technizität selbst entstehen, und den Werten, die durch gesellschaftliche Prozesse entstehen, unterschieden werden kann und muss. Die Art und Weise, wie technische Objekte gestaltet, hergestellt und benutzt werden, bestimmt die Form der Gesellschaft mit. Der Zugang über die Gesten erlaubt es, das Selbstverhältnis des Menschen zu seiner sozio-technischen Umwelt in unterschiedlichen Tiefenschärfen zu betrachten. Im gleichen Maße, wie Praxis und Operationalität in den Vordergrund rücken, werden die Gesten als Möglichkeiten der Teilhabe an der sozio-technischen Umwelt von größerer Bedeutung.

Dies führt letztlich dazu, dass die Frage, welche Objekte Teilhabe erlauben und in welchem Maße, einen höheren Stellenwert gewinnt (Schick im Erscheinen). Das „Denken mit den Händen“ fordert letztlich dazu auf, auch die Operationen, die auf den Mikrochips ablaufen, während man smart devices benutzt, wenn schon nicht $\mathrm{zu}$ verstehen, so immerhin mitzudenken. Man hält sie ja schließlich in Händen.

\section{Literatur}

Barthélémy, Jean-Hugues (2008): D’une recontre fertile de Bergson et de Bachelard, in: Worms, Frédéric/Wunenburger, Jean-Jacques (Hg.): Bachelard et Bergson, continuité et discontinuité?, Paris, 223-238.

Bergson, Henri (2013): Schöpferische Evolution, hrsg. V. Rémi Brague, übers. v. Margarethe Drewsen, Hamburg.

Bergson, Henri (1933): Die beiden Quellen der Moral und der Religion, übers. v. Eugen Lerch, Jena. 
Charrié, Pierre (2008): La Beauté Du Geste et La Machine (Mémoire de Fin d’études), Ensci-Les Ateliers.

Guillerme, André (2017): De Diderot à Taylor: exprimer les techniques du geste, in: Bouillon, Didier/Guillerme, André/Mille, Martine/Piernas, Gersende (Hg.): Gestes techniques, techniques du geste. Approches pluridisciplinaires, Villeneuve d'Ascq, 449-462.

Haudricourt, André-Georges (1987): La Technologie Science Humaine. Recherches d'histoire et d'ethnologie des Techniques, Paris.

Heilmann, Till (2016): Zur Vorgängigkeit Der Operationskette in Der Medienwissenschaft und bei Leroi-Gourhan, in: Internationales Jahrbuch für Medienphilosophie 2/1, 7-29.

Heilmann, Till (2017): Der Klang der breiten Rille, in: Internationales Jahrbuch für Medienphilosophie 3/1, https://jbmedienphilosophie.de/2017/3-replik-heilmann/.

Hussain, Shumon T. (2018): Kreativität, Technizität und Autopoiesis - Zur Bedeutung des Homo faber für das Verständnis der frühsten Menschheitsgeschichte, in: Zeitschrift für Kulturwissenschaften 4 (2): 49-66.

Ingold, Timothy (2011): Being Alive. Essays on Movement, Knowledge and Description, London/New York.

Kapp, Ernst (2015): Grundlinien Einer Philosophie Der Technik: Zur Entstehungsgeschichte der Kultur aus neuen Gesichtspunkten, Hamburg.

Latour, Bruno (1994): On Technical Mediation: Philosophy - Sociology - Genealogy, in: Common

Knowledge 3/2, 29-64.

Leroi-Gourhan, André (1943): Évolutions et techniques: L'homme et la matière, Paris.

Leroi-Gourhan, André (1945): Évolutions et techniques: Milieu et technique, Paris.

Leroi-Gourhan, André (2000): Hand und Wort: die Evolution von Technik, Sprache und Kunst, übers. v. Michael Bischoff, Frankfurt am Main.

Loeve, Sacha/Guchet, Xavier/Bensaude Vincent, Bernadette (Hg.) (2018): French Philosophy of Technology, Philosophy of Engineering and Technology, 29. Berlin/Heidelberg/New York.

Malafouris, Lambros (2013): How Things Shape the Mind: A Theory of Material Engagement, Cambridge, Massachusetts.

Malafouris, Lambros (2015): Metaplasticity and the Primacy of Material Engagement, in: Time and Mind 8/4, 351-371.

Mauss, Marcel (1933): Marcel Mauss, Discussion Avec Jean Piaget, in: Jean Piaget (Hg.): L'indivdualité en histoire. L'individu et la Formation du Raison, Paris, 118-121.

Mauss, Marcel. (2010): Die Techniken des Körpers, in: Marcel Mauss (Hg.): Soziologie und Anthropologie 2, übers. v. Henning Ritter, München, 197-220.

Mauss, Marcel (2015): Auffassungen, die dem Begriff der Materie vorausgegangen sind, übers. v. Johannes F. M. Schick, in: Zeitschrift für Kulturwissenschaften: Begeisterung und Blasphemie 2/2015, 233-238.

Mersch, Dieter (2016): Kritik Der Operativität. Bemerkungen Zu Einem Technologischen Imperativ, in: Internationales Jahrbuch Für Medienphilosophie 2/1, 31-52.

Parrochia, Daniel (2009): French Philosophy of Technology, in: Brenner, Anastasios/Gayon, Jean (Hg.): French Studies in The Philosophy Of Science: Contemporary Research in France, Dordrecht, 51-70.

Schick, Johannes F. M. (2018): Mechanik, Mystik und stumme Intelligenz: Doch eine Homo faber- Story, in: Zeitschrift für Kulturwissenschaften: Homo Faber 2/2018, 67-81. 
Schick, Johannes F. M. (2019): Towards an Interdisciplinary Anthropology? The Transformative Epistemologies of Bergson, Bachelard and Simondon, in: Parrhesia: A Journal of Critical Philosophy 31, 103-135. http://www.parrhesiajournal.org/parrhesia31/parrhesia31_ schick.pdf.

Schick, Johannes F. M. (im Erscheinen): The Potency of Open Objects. (Re-)Inventing New Modes of Being Human with Henri Bergson, Franco „Bifo“ Berardi and Gilbert Simondon, in: Techné: Research in Philosophy and Technology.

Scholz, Trebor (2017): Uberworked and Underpaid: How Workers Are Disrupting the Digital Economy, Cambridge/Malden.

Schüttpelz, Erhard (2006): Die medienanthropologische Kehre der Kulturtechniken, in: Engell, Lorenz/Siegert, Bernhard/Vogl, Joseph (Hg.): Kulturgeschichte als Mediengeschichte (Oder Vice Versa?), Archiv für Mediengeschichte, Weimar, 87-110.

Schüttpelz, Erhard (2017): Die Erfindung der Twelve-Inch. Der Homo Sapiens und Till Heilmanns Kommentar zur Priorität der Operationskette, in: Internationales Jahrbuch Für Medienphilosophie 3/1, 217-234.

Schüttpelz, Erhard (im Erscheinen): Vom Werkzeug zum Behälter, vom Behälter zum Medium: Die Ausweitungen des Körpers, in: Engelhardt, Nina/Schick, Johannes F. M. (Hg.): Erfinden, Schöpfen, Machen: Körpertechniken - Imaginationstechniken, Bielefeld.

Sigaut, François (1994): Technology, in: Ingold, Thimothy (Hg.): Companion Encyclopedia of Anthropology, London/ New York, 420-459.

Sigaut, François (2012): Comme homo devint faber, Paris.

Sigaut, François (2018): Die Homo Faber Kontroverse, übers. v. Schick, Johannes F. M., in: Zeitschrift für Kulturwissenschaften: Homo Faber 2/2018,17-33.

Simondon, Gilbert (2005): L'individuation à la lumière des notions de forme et d'information, Grenoble.

Simondon, Gilbert (2012): Die Existenzweise technischer Objekte, Zürich.

Simondon, Gilbert (2014): Sur la technique (1953-1983), Paris.

Simondon, Gilbert (2014a): Psychosociologie de la technicité, in: Simondon, Gilbert: Sur la technique (1953-1983), Paris, 27-129.

Simondon, Gilbert (2014b): Place d'une initiation technique dans une formation humaine complète (1953), in: Simondon, Gilbert: Sur la technique (1953-1983), Paris, $203-232$.

Simondon, Gilbert (2014C): Prolégomènes à une refonte de l'enseignement (1954), in: Simondon, Gilbert: Sur la technique (1953-1983), Paris, 233-253.

Zuboff, Shoshana (2019): The Age of Surveillance Capitalism: The Fight for the Future at the New Frontier of Power, London. 